\title{
PROCESOS DE EROSIÓN EN LOS BADLANDS ALICANTINOS
}

\author{
A. Cerdà i Bolinches* \\ R. Navarro i Moreno
}

\section{RESUMEN}

Los procesos de erosión son muy activos en la provincia de Alicante debido a las reducidas e irregulares precipitaciones, al substrato altamente erosionable y al milenario uso antrópico. Estas condiciones dan lugar a la formación de zonas acarcavadas o badlands, los cuales contribuyen con elevados volúmenes de agua y sedimentos a los cauces. A partir de experimentos con lluvia simulada se estudian los procesos de erosión y se cuantifican sus tasas a escala de entre-regueros $\left(<1 \mathrm{~m}^{2}\right)$ en dos zonas de badlands típicos del territorio alicantino: Monnegre y Petrer. En general, las tasas de erosión y escorrentía son muy elevadas, lo que se debe al rápido encharcamiento y la inmediata formación de la escorrentía superficial con la lluvia. La zona de Monnegre presenta menores pérdidas de agua, suelo y solutos que la de Petrer, aunque las diferencias dentro de cada zona son mayores que entre ellas. La inexistencia de cubierta vegetal y fragmentos de roca da lugar a altas tasas de escorrentía (54\%) y concentración de sedimentos que oscilan entre 20 y $82 \mathrm{~g} \mathrm{l}^{-1}$, por lo que las tasas de erosión son muy altas $\left(1,5 \mathrm{Kg} \mathrm{m}^{2} \mathrm{~h}^{-1}\right)$. La pendiente es el factor más importante de las tasas de erosión en los badlands, a lo que hay que sumar el control químico de los procesos de erosión.

Palabras clave: Badlands, Erosión, Hidrología, Lluvia simulada.

\section{ABSTRACT}

The erosional processes are very active in the province of Alicante (SE Spain) due to the low and irregular precipitations, to the highly erodible lithology and the continual human landuse. The conditions result in the formation of gully areas or badlands which contribute with high runoff volumes and sediments to the rivers. By means of simulated rainfall experiments the erosional proceses are studied and the erosion rates measured at inter-rill scale $\left(<1 \mathrm{~m}^{2}\right)$ in two typical badland sites of the Alicante territory: Monnegre and Petrer. In general, the erosion and runoff rates are very high, which is due to the quick ponding and the fast formation of the surface runoff with the rainfall. The Monnegre site had lower water, soil and solute losses than the Petrer site although the differences within each site are greater than between sites. The negligible vegetation cover and

* Departamento de Geografía. Universidad de Valencia. 
the rock fragment cover result in high runoff rates $(54 \%)$ and sediment concentration ranging from 20 to $82 \mathrm{~g} \mathrm{l}^{-1}$, and as a consequence very high erosion rates $\left(1,5 \mathrm{Kg} \mathrm{m}^{2} \mathrm{~h}^{-1}\right)$. Moreover the chemical control on the erosional processes the slope angle is the main factor controlling the erosion rates $(54 \%)$ on the badland landscapes.

Key words: Badlands, Erosion, Hydrology, Simulated Rainfall.

\section{Introducción}

\subsection{Factores ambientales y erosión en la provincia de Alicante}

Alrededor del $60 \%$ del territorio de la provincia de Alicante recibe precipitaciones medias anuales inferiores a $400 \mathrm{~mm}$, y un $30 \%$ no supera los $300 \mathrm{~mm}$. Incluso, en uno de cada cuatro años las precipitaciones no superan los $300 \mathrm{~mm}$ anuales en más del $70 \%$ del territorio, y en una parte importante del sur de la provincia es inferior a $200 \mathrm{~mm}$ (PÉREZ CUEVA, 1994). Desde el punto de vista litológico, en la provincia de Alicante existen materiales poco erosionables como son las calizas, areniscas y las dolomías, principalmente en la franja norte montañosa. Sin embargo, los materiales más extendidos (> 60\%) son las arcillas y yesos del Keuper, las margas de distintos períodos (Cretácico, Paleógeno, Neógeno, etc.) y los depósitos cuaternarios donde arcillas, limos, arenas, gravas y conglomerados poco cementados son abundantes (MATARREDONA y MARCO, 1991). En el territorio alicantino, el milenario uso del suelo por distintas civilizaciones ha dado lugar a la alteración de la cubierta vegetal de forma que la composición florística ha sido modificada de una forma substancial (COSTA, 1986). Ello a su vez ha favorecido la degradación de los suelos y el inicio de procesos de erosión acelerada que en algunos casos pueden ser considerados como parte del proceso de desertificación que sufre el Mediterráneo y en general las zonas semiáridas (PUIGDEFÁBREGAS, 1996).

La explotación antrópica del medio, reducidas precipitaciones y roquedos muy erosionables conforman la realidad ambiental presente en la provincia de Alicante, la cual no es diferente de otros paisajes del Mediterráneo Español (LÓPEZ BERMÚDEZ y ALBADALEJO, 1990). Bajo estas condiciones los procesos de erosión acelerada se hacen evidentes con la formación de pequeñas cárcavas, las cuales se pueden transformar en un campo de cárcavas más amplio en situaciones muy concretas (Desierto de Tabernas y Bardenas Reales en la Península Ibérica). En Alicante esta situación no es tan extendida, pero sí existen zonas donde la presencia de badlands es importante (HARVEY y CALVO, 1989).

\subsection{Los badlands}

Los badlands son paisajes en los que la vegetación es reducida o inexistente, donde los suelos consisten en una capa de regolita de pocos centímetros de profundidad que hacen inviables las actividades agrícolas debido a la activa dinámica de los procesos geomórficos superficiales y a sus geoformas (BRYAN y YAIR, 1982). Estos ambientes son los que aportan gran parte de los sedimentos y escorrentías a los cauces, siendo en gran medida los responsables de inundaciones, aterramiento de embalses, roturas de obras de infraestructura y pérdida de bienes materiales y vidas humanas debido a los elevados caudales generados (CAMPBELL, 1974). A pesar de ello es poco conocido el funcionamiento de estas superficies durante la lluvia, lo cual es fundamental para prevenir y predecir riesgos naturales como son la erosión acelerada que afecta a la propia zona y las inundaciones 
existentes en las partes bajas de las cuencas de drenaje. También es importante el estudio de los badlands para conocer el funcionamiento de los sistemas geomórficos (GREGORY y WALLING, 1973), ya que en estos geosistemas, los procesos superficiales son muy activos en espacios muy reducidos y pueden ser utilizados como laboratorios geomorfológicos en miniatura (ABRAHAMS y PARSONS, 1994). De hecho, la actividad de la arroyada superficial difusa da lugar a la formación de regueros — formas propias de la escorrentía superficial concentrada- en recorridos menores a 1 metro.

Los badlands se originan sobre roquedos muy erosionables y en zonas semiáridas, donde la precipitación es escasa e irregular (CAMPBELL, 1989). Además, cuando la presencia de actividad antrópica favorece alteraciones importantes de la cubierta edáfica y vegetal, y por lo tanto en el régimen hídrico de los suelos y las cuencas de drenaje, los paisajes de badlands se generalizan (HOWARD, 1994). En el caso de Alicante las tres condiciones apuntadas se cumplen, y por ello existe una importante presencia de zonas desnudas donde los procesos erosivos son muy activos.

En ambientes semiáridos las precipitaciones —además de por su escasez- se caracterizan por su irregularidad, lo que hace que el estudio de los procesos hidrogeomorfológicos sea muy complicado y costoso. Una opción desarrollada por hidrólogos, geomorfólogos y agrónomos para el estudio de estos ambientes ha sido el desarrollo de simuladores de lluvia, con los cuales se consigue mayor precisión en las mediciones (MEYER, 1994). Estos instrumentos permiten estudiar los procesos de erosión de forma muy detallada al poder conocer y cuantificar las variables que influyen sobre ellos: humedad, costras, pedregosidad, etc., y detallar sus cambios a lo largo del evento de lluvia.

\subsection{Objetivos}

En este trabajo se estudian los procesos de erosión en dos zonas de badlands típicas de la provincia de la Alicante: Petrer y Monnegre (ver figuras 1 y 2). Para acelerar la toma de datos se realizaron experimentos con lluvia simulada a una intensidad elevada $\left(55 \mathrm{~mm} \mathrm{~h}^{-1}\right)$ con el fin de conocer la respuesta erosiva e hidrológica de los suelos ante eventos de reducida frecuencia pero alta magnitud, los cuales son fundamentales para entender los procesos geomorfológicos y los paisajes desarrollados en ambientes semiáridos (THORNES, 1976). En concreto, esta investigación se realiza a escala de entre-regueros para caracterizar la forma de generarse la escorrentía y conocer los procesos y tasas de erosión.

\section{Zonas de estudio y características de los suelos}

Las dos zonas de estudio seleccionadas, presentan suelos desnudos donde los regueros, las costras y las grietas son las formas superficiales más evidentes (Fig. 1). La zona de Petrer $\left(8^{\circ} 28^{\prime} \mathrm{N} ; 0^{\circ} 45^{\prime} \mathrm{W}\right)$, situada al noreste de esta ciudad corresponde a una cuenca de orden uno, tributaria del Barranco de los Molinos, el cual a su vez lo es del Riu Vinalopó por la izquierda. El roquedo son margas cretácicas aunque también existen badlands sobre los depósitos arenosos del glacis pleistoceno (IGME, 1978a). Las cárcavas se han formado por la incisión del anterior valle pleistoceno debido al diapirismo de la zona y al abancalamiento y posterior abandono del fondo de valle (RODRÍGUEZ AIZPEOLEA, 1992). Las condiciones climatológicas se caracterizan por la baja precipitación media anual: de 296 a 339 mm según autores (GEIGER, 1970; PAYÀ y CERDÀ, 1992). Una recopilación posterior - más completa y exhaustiva - atribuye $334 \mathrm{~mm}$ al cercano observatorio de Monòver (PÉREZ CUEVA, 1994). La temperatura media anual es de $16^{\circ} \mathrm{C}$, con un máximo de $23,3^{\circ} \mathrm{C}$ en julio y $6,6^{\circ} \mathrm{C}$ en enero. 

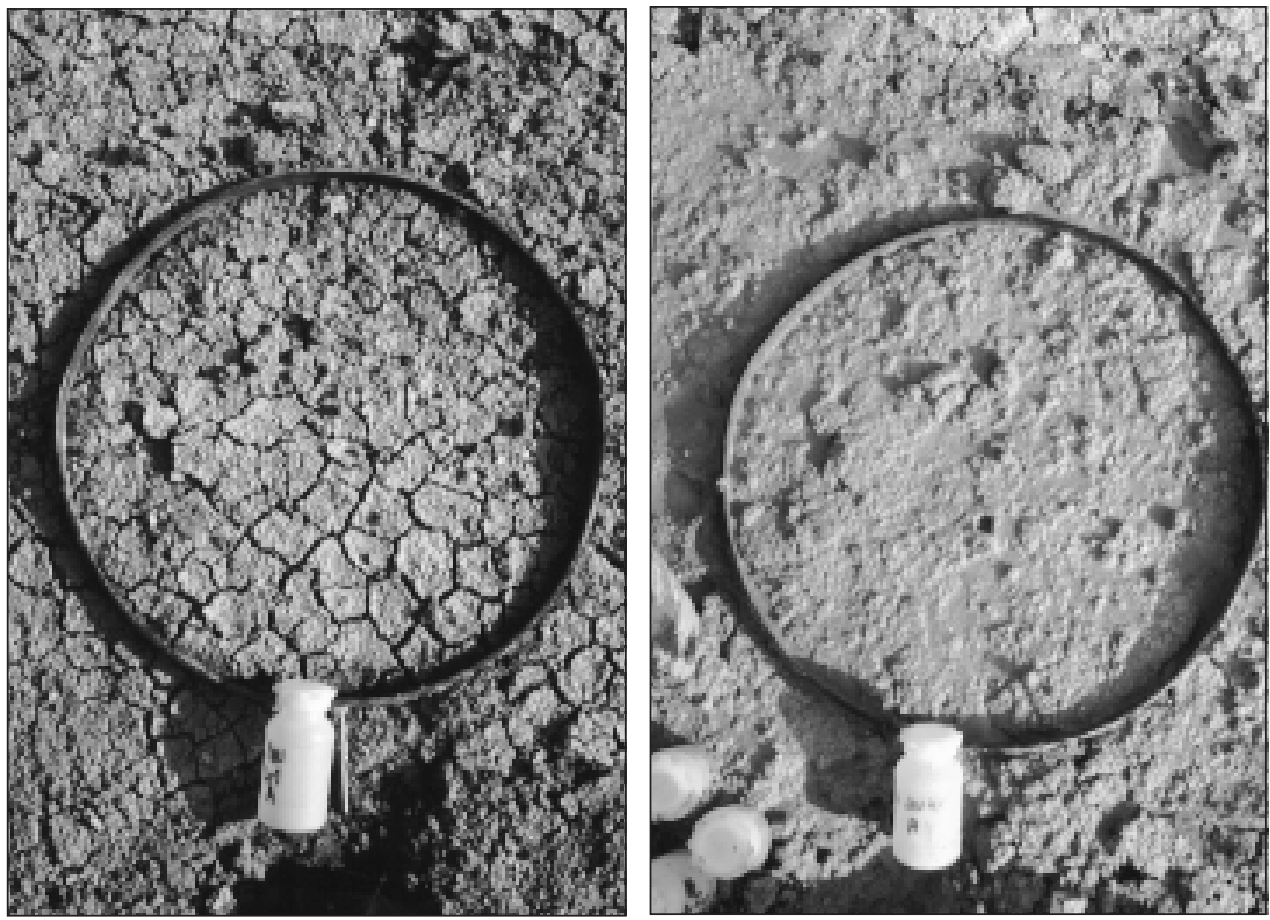

Figura 1. Parcela de simulación de lluvia sin reguero: a, antes de la lluvia; b, después de la lluvia.

La zona de Monnegre ( $38^{\circ} 27^{\prime} \mathrm{N}$; $\left.0^{\circ} 30^{\prime} \mathrm{W}\right)$, se encuentra junto al río del mismo nombre entre las ciudades de Xixona y Muxtamel y en ella se han estudiado los suelos situados en distintas cárcavas tributarias por la margen derecha del río. El roquedo son margas senonienses, aunque también aquí los badlands se han desarrollado sobre arcillas del Keuper y sedimentos pleistocenos (CERDÀ, en preparación). La zona se caracteriza por la existencia de diferentes terrazas fluviales, con una evolución cuaternaria muy rápida fruto de la fuerte incisión del Riu Monnegre favorecida sin duda por la dinámica diapírica de la zona (IGME, 1978b). La incisión de la red de drenaje ha favorecido el desmantelamiento de los depósitos de ladera y fluviales, dejando materiales más erosionables como las margas Senoniense en superficie. Las temperaturas medias mensuales, tanto la máxima como la mínima, son muy altas (enero: $11,6^{\circ}$; agosto: $25,5^{\circ}$, para el cercano observatorio de Alicante, Ciudad Jardín), mientras que la precipitación media anual son también reducidas (274 mm en el aeropuerto del Altet y $325 \mathrm{~mm}$ para el observatorio Ciudad Jardín).

En Petrer, las laderas son cortas $(40-50 \mathrm{~m})$ y sus pendientes son muy elevadas $\left(35-45^{\circ}\right)$, mientras que en Monnegre, a pesar de la incisión del río las pendientes medias son menores $\left(20-25^{\circ}\right)$. Los suelos se caracterizan en ambos casos por la práctica inexistencia de materia orgánica, elevado porcentaje de la fracción limo (50-60\%), alto contenido en carbonato cálcico (60-70\%) y densidad aparente muy elevada (1,40-1,60 $\left.\mathrm{g} \mathrm{cm}^{-3}\right)$. La inexistencia de vegetación, junto a las anteriores características, hace que la existencia de costras sea un factor muy importante en la explicación del comportamiento hidrológico-erosivo de estos suelos, como también lo es la existencia de grietas y patrones dobles de éstas en forma de polígonos, sobre todo en los suelos menos desarrollados y con mayores contenidos en 
MEDIA, DESVIACIÓN ESTÁNDAR Y COEFICIENTE DE VARIACIÓN DE LA PENDIENTE, PROFUNDIDAD DEL SUELO Y CUBIERTA DE FRAGMENTOS DE ROCA DE LAS PARCELAS ESTUDIADAS EN PETRER Y MONNEGRE

\begin{tabular}{|c|c|c|c|}
\hline Prueba & $\begin{array}{c}\text { Pendiente } \\
\left({ }^{\circ}\right)\end{array}$ & $\begin{array}{l}\text { Profundidad del suelo } \\
\text { (cm) }\end{array}$ & $\begin{array}{c}\text { Fragmentos de roca } \\
(\%)\end{array}$ \\
\hline \multicolumn{4}{|l|}{ Monnegre } \\
\hline 1 & 28 & 4 & 3 \\
\hline 2 & 8 & 3 & 13 \\
\hline 3 & 30 & 2 & 12 \\
\hline 4 & 20 & 2 & 0 \\
\hline 5 & 18 & 6 & 0 \\
\hline 6 & 14 & 2 & 14 \\
\hline 7 & 12 & 2 & 10 \\
\hline 8 & 15 & 2 & 3 \\
\hline 9 & 24 & 4 & 2 \\
\hline 10 & 10 & 7 & 4 \\
\hline Media & 17,90 & 3,40 & 6,10 \\
\hline std & 7,52 & 1,84 & 5,53 \\
\hline $\operatorname{cv}(\%)$ & 42,01 & 54,06 & 90,60 \\
\hline \multicolumn{4}{|l|}{ Petrer } \\
\hline 1 & 8 & 5 & 12 \\
\hline 2 & 30 & 3 & 0 \\
\hline 3 & 8 & 3 & 14 \\
\hline 4 & 14 & 2 & 5 \\
\hline 5 & 40 & 3 & 2 \\
\hline 6 & 40 & 3 & 2 \\
\hline 7 & 40 & 2 & 2 \\
\hline 8 & 34 & 2 & 5 \\
\hline 9 & 30 & 2 & 9 \\
\hline 10 & 34 & 2 & 5 \\
\hline Media & 27,80 & 2,70 & 5,60 \\
\hline std & 12,94 & 0,95 & 4,65 \\
\hline $\operatorname{cv}(\%)$ & 46,56 & 35,14 & 82,99 \\
\hline
\end{tabular}

arcilla (Figs. 1 y 1a). La estructura de estos suelos es débil, con consistencia dura en seco y friable/muy friable en húmedo, y muy adherente y plástica en mojado. Aunque los suelos pueden ser clasificados como regosoles, en realidad son un espesor variable de regolita.

En la tabla 1 se presentan algunos datos de las características de las parcelas estudiadas. La pendiente es elevada en las dos zonas de estudio: $18^{\circ}$ en Monnegre y $28^{\circ}$ en Petrer de media. Estas diferencias son debidas a que la zapa basal de los campos de cultivos en Petrer ha favorecido laderas con pendientes mayores. Las variaciones de pendiente entre parcelas es semejante en ambas zonas (coeficiente de variación de 42 y 47\%), oscilando entre $8^{\circ}$ y $30^{\circ}$ en Monnegre; y entre $8^{\circ}$ y $40^{\circ}$ en Petrer.

La profundidad del suelo es de unos $3 \mathrm{~cm}$ (3,4 cm en Monnegre y 2,7 cm en Petrer). En el primer caso oscila entre 2 y $7 \mathrm{~cm}$ y en el segundo entre 2 y $5 \mathrm{~cm}$. Los coeficientes de variación son semejantes entre las dos zonas de estudio: 54\% en Monnegre y 35\% en Petrer. La cubierta de fragmentos de roca, aunque reducida es importante para entender los procesos de erosión, especialmente en suelos donde no existe vegetación que proteja los suelos. En este aspecto, Monnegre presenta valores ligeramente mayores $(6,1 \%)$ que Petrer 
(5,6\%), oscilando en ambos casos entre 0 y $13 \%$. El coeficiente de variación en ambos casos es semejante (90 y $83 \%$ respectivamente).

Las grietas alcanzan anchuras de hasta $1 \mathrm{~cm}$ (de 4 a $10 \mathrm{~mm}$ ) y forman polígonos de 5-10 cm de anchura. Estas grietas tienen profundidades de 3-4 cm y en ocasiones están conectadas a la red de fracturas de la roca madre. También existe una segunda red de grietas de menor anchura $(1 \mathrm{~mm})$ y profundidad $(1-3 \mathrm{~mm})$ aunque no llegan a formar una red de polígonos permanente. Las morfologías de los suelos son muy variables dependiendo de la pendiente, de las diferencias litológicas, exposición, etc. (PAYÀ y CERDÀ, 1992; CALVO et al., 1991; 1992; CERDÀ y GARCÍA FAYOS, en prensa a; en prensa b).

\section{Métodos}

En este trabajo se aborda el estudio de los procesos de erosión a escala menor a $1 \mathrm{~m}^{2}$., es decir, las superficies entre-regueros. Aunque en ocasiones se han realizado mediciones en superficies afectadas por la formación de regueros, nunca se ha abarcado la cuenca de drenaje de un reguero en su totalidad, lo que supondría realizar la medición mediante parcelas mayores a $10 \mathrm{~m}^{2}$. Los experimentos han sido llevados a cabo en parcelas de $0,24 \mathrm{~m}^{2}$. mediante lluvia simulada aplicada sobre $1 \mathrm{~m}^{2}$. con el fin de eliminar el efecto del borde de parcela. Para ello se ha utilizado un simulador de lluvia portátil, el cual fue diseñado para su uso en laderas con fuertes pendientes como las de los badlands (CERDÀ, 1995a).

Todos los experimentos se realizaron durante el máximo período de sequedad en la zona (julio y agosto) por lo que los resultados muestran la respuesta hidrológica y erosiva de los suelos cuando las grietas alcanzan su máxima amplitud, el suelo está más seco y también cuando más material está disponible de ser erosionado por la inexistencia de precipitaciones previas que lavaran la superficie de sedimentos. La duración de cada chaparrón fue de 40 minutos a una intensidad de $55 \mathrm{~mm} \mathrm{~h}^{-1}$. El período de retorno de estos chaparrones es de 7-8 años en la zona de estudio (ELÍAS y RUIZ, 1979), es decir eventos de alta magnitud y reducida frecuencia.

Para conocer la curva de escorrentía completa se tomaron muestras del caudal cada 30 ó 60 segundos como se explica en CERDÀ (1993b). Al menos cinco veces se tomaron muestras para determinar la concentración de sedimentos (por desecación) y las tasas de erosión, aunque en ocasiones se tomaron hasta 30 muestras para verificar la validez de los datos (Fig. 2). En tres casos la concentración de sedimentos de la escorrentía (Cs) se muestreó todo el caudal a intervalos de un minuto con el fin de conocer con mayor precisión los cambios temporales en la erodibilidad de los suelos. Los 40 minutos de duración de los experimentos fueron suficientes para alcanzar las tasas de escorrentía final estable como se demuestra en diversos trabajos (CERDÀ, 1993a) (Fig. 2). También se midió la conductividad eléctrica de la escorrentía $(\mathrm{Sa})$ en el campo la cual permite conocer la salinidad de la escorrentía (NAVAS, 1988). Tanto la $C s$ como la $S a$ muestra cambios a lo largo del experimento. Para conocer su tendencia - a crecer o decrecer - se ha ajustado una ecuación lineal $(\mathrm{y}=\mathrm{a}+\mathrm{b}(\mathrm{x}))$, donde el parámetro $\mathrm{b}(+\mathrm{b}$ o $-\mathrm{b})$ indica si la tendencia es al aumento (+b) o al decrecimiento (-b) de la $C s(T c s)$ o de $S a(T s a)$.

El tiempo de encharcamiento ( $t p)$ (40\% de la superficie está encharcada) y el inicio de la escorrentía en superficie (te) (movilización del encharcamiento) se determinó de forma visual. La llegada de la escorrentía a la salida o inicio del hidrograma (tec) se anotó cuando se inició el goteo en el colector, lo que equivale al inició del hidrograma. La curva de escorrentía/infiltración se modeliza según la ecuación de HORTON (1940) como se explica en CERDÀ (1993a) (Fig. 2): 


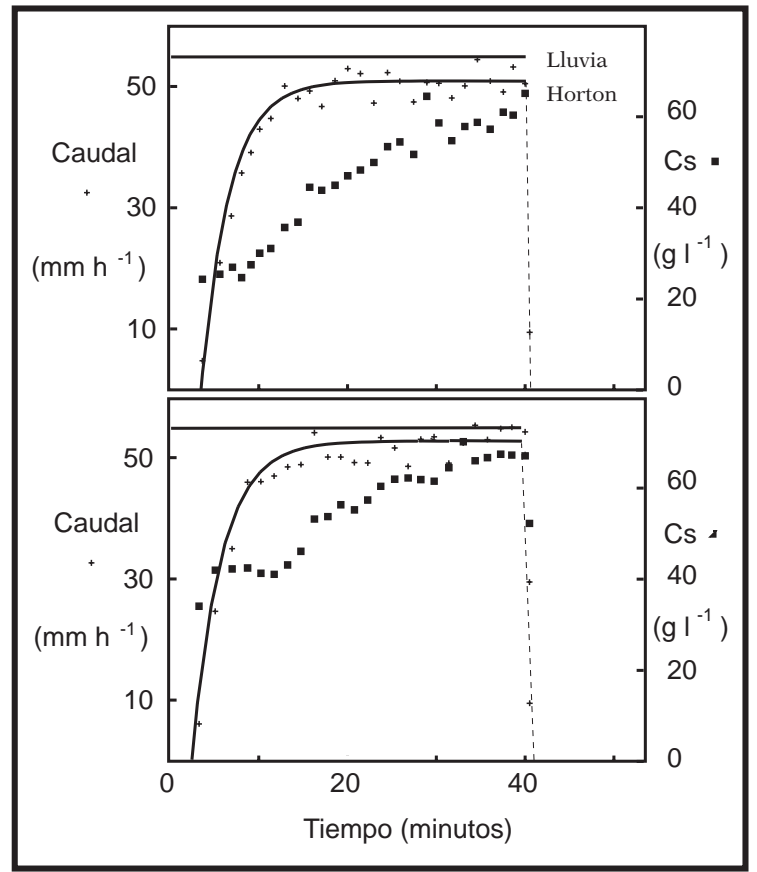

FIGURA 2. Ejemplo de las mediciones realizadas. Escorrentía (+), concentración de sedimentos (n), precipitación y ajuste de la ecuación de Horton a las tasas de escorrentía.

$$
f=f c+(f o-f c) e^{\alpha t}
$$

Donde:

$f=$ tasa de infiltración instantánea

$f c=$ tasa de infiltración final estable

$f o=$ tasa de infiltración inicial $(t=0)$

$\alpha=$ constante empírica (forma de la curva de infiltración)

\section{Resultados y discusión}

Los experimentos se realizaron durante el verano de 1991 por lo que la humedad previa de los experimentos es insignificante (Tabla 2). La humedad superficial $(0-2 \mathrm{~cm})$ en Monnegre fue de 0,99\% mientras que en Petrer es ligeramente mayor (1,45\%). A 4-6 cm las diferencias son incluso menores $(2,6$ y $2,9 \%)$, y en valores medios $(0-6 \mathrm{~cm})$ las diferencias son también poco importantes $(1,8-2,2 \%)$. Las variaciones espaciales -expresadas como coeficientes de variación- oscilan entre el 39 y el $21 \%$ en Monnegre y 35 y $37 \%$ en Petrer respectivamente para los horizontes superficiales $(0-2 \mathrm{~cm})$ y subsuperficiales (4-6 cm). Estos datos confirman la sequedad extrema de los suelos antes de los experimentos, así como su reducida variación espacial durante los períodos secos, comportamiento muy distinto al encontrado durante las estaciones húmedas (CERDÀ, 1996a). 
La lluvia origina cambios drásticos y rápidos en la superficie de los badlands, y ello da lugar a la formación de volúmenes de escorrentía importantes. Como consecuencia del impacto de las gotas de lluvia, el primer efecto de la lluvia es el splash, aumentando la capacidad erosiva de las gotas con el incremento de la humedad del suelo y su encharcamiento (SAVAT, 1981). El encharcamiento en la superficie del suelo aparece casi inmediatamente: 1' 46" en Monnegre y 1' 52" en Petrer en término medio. Los valores oscilan entre 0' 36" y 2' 30" en Monnegre; y 0' 50" y 2' 40" en Petrer, con un coeficiente de variación del $39 \%$.

El inicio de la escorrentía en la salida de la parcela $(t e)$ - inicio del hidrograma- se produce antes en Monnegre: entre 1' 40" y 5' 10" (3' 22" de media), que en Petrer: entre

Tabla 2

HUMEDAD PREVIA DE LOS SUELOS A DISTINTAS PROFUNDIDADES: 0-2, 4-6 CM Y MEDIA ENTRE AMBAS. CAMBIOS PRODUCIDOS POR LA LLUVIA: TIEMPO DE ENCHARCAMIENTO (tp), TIEMPO DE INICIO DE LA ESCORRENTÍA EN SUPERFICIE (te), TIEMPO DE INICIO DE LA ESCORRENTÍA EN EL COLECTOR (tec), TIEMPO NECESARIO PARA PRODUCIRSE EL CIERRE DE LAS GRIETAS (tcg) Y VOLUMEN DE LLUVIA NECESARIO PARA GENERARSE ESCORRENTÍA SUPERFICIAL (Ve)

\begin{tabular}{|c|c|c|c|c|c|c|c|c|}
\hline \multirow[b]{2}{*}{ Prueba } & \multicolumn{3}{|c|}{ Humedad previa } & \multicolumn{5}{|c|}{ Cambios producidos por la lluvia } \\
\hline & $\begin{array}{c}(0-2 \mathrm{~cm}) \\
(\%)\end{array}$ & $\begin{array}{c}(4-6 \mathrm{~cm}) \\
(\%)\end{array}$ & $\begin{array}{c}\text { Media } \\
(\%)\end{array}$ & $t p$ & te & tec & tcg & $\begin{array}{c}V e \\
(\mathbf{m m})\end{array}$ \\
\hline Monnegre & & & & & & & & \\
\hline 1 & 1,4 & 3,0 & 2,2 & $1^{\prime} 20 "$ & 1' 40" & 4' $00 "$ & 9' 50" & 1,28 \\
\hline 2 & 1,1 & 3,1 & 2,1 & $1^{\prime} 40^{\prime \prime}$ & $2^{\prime} 52^{\prime \prime}$ & 6'00" & $200^{\prime} 00^{\prime \prime}$ & 2,31 \\
\hline 3 & 1,4 & 2,5 & 2,0 & $2^{\prime} 30^{\prime \prime}$ & 5' $03 "$ & 6' $02^{\prime \prime}$ & $12^{\prime} 00^{\prime \prime}$ & 4,61 \\
\hline 4 & 1,0 & 2,8 & 1,9 & $2^{\prime} 05^{\prime \prime}$ & 3' $00^{\prime \prime}$ & $8^{\prime} 00^{\prime \prime}$ & $17^{\prime} 00^{\prime \prime}$ & 2,75 \\
\hline 5 & 1,2 & 2,1 & 1,7 & $1^{\prime} 30^{\prime \prime}$ & $1^{\prime} 40 "$ & $2^{\prime} 30^{\prime \prime}$ & $7^{\prime} 10^{\prime \prime}$ & 1,28 \\
\hline 6 & 1,2 & 3,1 & 2,2 & 0' 36" & $2^{\prime} 30^{\prime \prime}$ & 6' 30" & 13'00" & 2,11 \\
\hline 7 & 1,1 & 3,0 & 2,1 & $1^{\prime} 20^{\prime \prime}$ & $2^{\prime} 44^{\prime \prime}$ & 7'00" & $15^{\prime} 00^{\prime \prime}$ & 2,24 \\
\hline 8 & 0,6 & 2,7 & 1,7 & $2^{\prime} 30^{\prime \prime}$ & 5' 10" & 6' $35^{\prime \prime}$ & 16' 30" & 4,68 \\
\hline 9 & 0,4 & 1,5 & 0,9 & $2^{\prime} 15^{\prime \prime}$ & 3' 30" & 6' $40^{\prime \prime}$ & $13^{\prime} 00^{\prime \prime}$ & 3,03 \\
\hline 10 & 0,4 & 2,1 & 1,3 & $1^{\prime} 50 "$ & $2^{\prime} 30^{\prime \prime}$ & 3'00" & 4' 30" & 2,11 \\
\hline Media & 0,99 & 2,60 & 1,79 & 1' $46^{\prime \prime}$ & $3^{\prime} 22^{\prime \prime}$ & $5^{\prime} 38^{\prime \prime}$ & $12^{\prime} 48^{\prime \prime}$ & 2,64 \\
\hline std & 0,39 & 0,55 & 0,42 & 0,62 & 1,30 & 1,82 & 4,75 & 1,19 \\
\hline $\operatorname{cv}(\%)$ & 39,19 & 21,11 & 23,68 & 39,44 & 45,02 & 32,93 & 37,32 & 45,02 \\
\hline Petrer & & & & & & & & \\
\hline 1 & 0,6 & 1,2 & 0,9 & $2^{\prime} 40^{\prime \prime}$ & $2^{\prime} 45^{\prime \prime}$ & 3'00" & 4' $30^{\prime \prime}$ & 2,25 \\
\hline 2 & 1,9 & 2,9 & 2,4 & 0' $50 "$ & 1' 10" & $2^{\prime} 00^{\prime \prime}$ & 7' 00" & 1,01 \\
\hline 3 & 1,2 & 2,1 & 1,7 & $2^{\prime} 00^{\prime \prime}$ & 3' 00" & 4' $00^{\prime \prime}$ & $7^{\prime} 25^{\prime \prime}$ & 2,75 \\
\hline 4 & 1,8 & 2,5 & 2,1 & $2^{\prime} 00^{\prime \prime}$ & $2^{\prime} 30^{\prime \prime}$ & 4' $00^{\prime \prime}$ & 5' 55" & 2,11 \\
\hline 5 & 1,3 & 2,6 & 1,9 & $1^{\prime} 00^{\prime \prime}$ & 1' 50" & $2^{\prime} 20^{\prime \prime}$ & $10^{\prime} 00^{\prime \prime}$ & 1,38 \\
\hline 6 & 1,3 & 2,6 & 1,9 & $1^{\prime} 35^{\prime \prime}$ & $2^{\prime} 02^{\prime \prime}$ & $2^{\prime} 20^{\prime \prime}$ & $10^{\prime} 41 "$ & 1,85 \\
\hline 7 & 2,3 & 4,5 & 3,4 & $2^{\prime} 40^{\prime \prime}$ & 3' 09" & 3' $30^{\prime \prime}$ & 3' $20^{\prime \prime}$ & 2,83 \\
\hline 8 & 1,3 & 2,7 & 2,0 & 1' 30" & 3' 00" & 3' 30" & 7' 05" & 2,75 \\
\hline 9 & 1,0 & 3,5 & 2,2 & $1^{\prime} 50 "$ & $2^{\prime} 00^{\prime \prime}$ & $2^{\prime} 25^{\prime \prime}$ & 4' $35 "$ & 1,83 \\
\hline 10 & 1,7 & 5,0 & 3,4 & $2^{\prime} 30^{\prime \prime}$ & $2^{\prime} 49^{\prime \prime}$ & $4^{\prime} 00^{\prime \prime}$ & $9^{\prime} 30^{\prime \prime}$ & 2,28 \\
\hline Media & 1,45 & 2,94 & 2,19 & 1' 52 " & $2^{\prime} 26^{\prime \prime}$ & 3'07" & 7' 00" & 2,10 \\
\hline std & 0,51 & 1,11 & 0,75 & 0,65 & 0,66 & 0,82 & 3,81 & 0,60 \\
\hline $\operatorname{cv}(\%)$ & 35,08 & 37,72 & 34,10 & 38,62 & 28,72 & 27,03 & 66,79 & 28,72 \\
\hline
\end{tabular}


1' 10" y 3' 09" (2' 26" de media). Las variaciones son menores en Petrer como lo demuestra el menor coeficiente de variación de te (29\%), frente al $45 \%$ de Monnegre. Las diferencias entre Monnegre y Petrer son insignificantes por lo que se refiere al encharcamiento $t p$, pero en Petrer se inicia la escorrentía más rápidamente, y ésta se produce con un volumen de lluvia menor (2,10 $\mathrm{mm}$ ) que en Monnegre $(2,64 \mathrm{~mm})$ (ver Tabla 2).

La escorrentía iniciada en la superficie alcanza con rapidez el colector de la parcela (de $55 \mathrm{~cm}$ de diámetro) debido a la inexistencia de vegetación y hojarasca, y por la reducida rugosidad de la superficie. En término medio, la escorrentía tarda 2' 16" en alcanzar el colector en Monnegre y tan sólo 41" en Petrer. Esta diferencias se ven reflejadas en los máximos y mínimos en cada zona de estudio para tec: 2' 30" - 8' en Monnegre y 2' - 4' en Petrer. Las variaciones espaciales son semejantes entre las dos zonas ( $c v: 33$ y $27 \%$ respectivamente).

El retraso en la formación de la escorrentía en Monnegre respecto a Petrer se debe a la menor tasa de infiltración de los suelos en esta última zona (Tabla 3) y al retraso en el cierre de las grietas. Este último aspecto $(t c g)$ muestra un comportamiento muy distinto en

Tabla 3

PRINCIPALES PARÁMETROS HIDROLÓGICOS. COEFICIENTE DE ESCORRENTÍA (Ce), TASA DE INFILTRACIÓN FINAL ESTABLE ( $f c$ ), VOLUMEN DE AGUA INFILTRADO DESPUÉS DE 30 MINUTOS (Vi30), Y COEFICIENTE DE ESCORRENTÍA ENTRE EL MINUTO O Y EL 15 (Ce15)

\begin{tabular}{|c|c|c|c|c|}
\hline Prueba & $C e$ & $\begin{array}{c}f c \\
\left(\mathbf{m m} \mathbf{h}^{-1}\right)\end{array}$ & $\begin{array}{c}V i 30 \\
(\mathrm{~mm})\end{array}$ & Ce15 \\
\hline \multicolumn{5}{|l|}{ Monnegre } \\
\hline 1 & 0,52 & 18,97 & 13,34 & 0,38 \\
\hline 2 & 0,41 & 21,75 & 16,67 & 0,21 \\
\hline 3 & 0,40 & 20,94 & 16,78 & 0,20 \\
\hline 4 & 0,42 & 18,58 & 16,50 & 0,17 \\
\hline 5 & 0,50 & 23,61 & 14,23 & 0,39 \\
\hline 6 & 0,46 & 18,85 & 15,27 & 0,25 \\
\hline 7 & 0,47 & 19,39 & 16,34 & 0,19 \\
\hline 8 & 0,43 & 20,71 & 15,95 & 0,23 \\
\hline 9 & 0,47 & 20,40 & 14,99 & 0,28 \\
\hline 10 & 0,52 & 23,32 & 13,66 & 0,43 \\
\hline Media & 0,46 & 20,65 & 15,37 & 0,27 \\
\hline std & 0,04 & 1,80 & 1,28 & 0,09 \\
\hline $\operatorname{cv}(\%)$ & 9,48 & 8,72 & 8,32 & 34,34 \\
\hline \multicolumn{5}{|l|}{ Petrer } \\
\hline 1 & 0,47 & 21,81 & 14,35 & 0,36 \\
\hline 2 & 0,68 & 6,85 & 8,43 & 0,53 \\
\hline 3 & 0,57 & 17,33 & 11,59 & 0,47 \\
\hline 4 & 0,60 & 14,23 & 11,36 & 0,44 \\
\hline 5 & 0,67 & 12,20 & 10,30 & 0,48 \\
\hline 6 & 0,71 & 10,25 & 9,34 & 0,51 \\
\hline 7 & 0,53 & 17,81 & 12,65 & 0,41 \\
\hline 8 & 0,71 & 7,43 & 7,44 & 0,59 \\
\hline 9 & 0,71 & 10,32 & 7,69 & 0,63 \\
\hline 10 & 0,60 & 9,38 & 10,55 & 0,42 \\
\hline Media & 0,62 & 12,76 & 10,37 & 0,48 \\
\hline std & 0,09 & 4,92 & 2,21 & 0,08 \\
\hline $\operatorname{cv}(\%)$ & 13,70 & 38,55 & 21,35 & 17,08 \\
\hline
\end{tabular}


Monnegre donde se produce con mayor retraso: 12' 48" (4' 30" - 20'), que en Petrer: 7' (4' 30" - 10' 41"), donde la grietas se cierran antes y favorecen la conformación de la escorrentía superficial más rápidamente (Tabla 2). El origen de estas diferencias en la respuesta hidrológica entre Petrer y Monnegre (te, $f c$ y $t c g$ ) está relacionado con la mayor pendiente de los suelos de Petrer. Así, a mayor pendiente la escorrentía se moviliza antes y alcanza el colector en poco tiempo. También la arroyada alcanza rápidamente las grietas, las satura de agua o las rellena de sedimentos, y con ello favorece su sellamiento, lo que favorece la rápida circulación del flujo superficial.

\subsection{Hidrología superficial}

Las características anteriormente comentadas sobre las superficies de los badlands (encostrados, alta densidad, agrietados, etc.) determinan encharcamientos y escorrentías muy rápidos. Después de 40 minutos de lluvia el caudal de la escorrentía es muy elevada. En Monnegre, el coeficiente de escorrentía fue del $46 \%$ (42-52\%) y en Petrer del $62 \%$ (47-7\%), con variaciones espaciales semejantes: 9,5 y $13,7 \%$ de $c v$ respectivamente.

El $\mathrm{Ce}$ durante los primeros 15 minutos de lluvia ( $\mathrm{Ce} 15)$ ilustra las elevadas tasas de escorrentía de los badlands ante volúmenes de lluvia reducido con respecto a otros suelos en ecosistemas mediterráneos (CERDÀ, 1995a), algunos de ellos situados en zonas muy cercanas a la zona de estudio (IMESON et al., 1996). En Monnegre, Ce15 es del 27\%, mientras que en Petrer es del 48\% (Tabla 3). Esta diferencia tan clara entre Monnegre y Petrer en los primeros 15 minutos de las tormentas se debe al retraso en la formación de la escorrentía en Monnegre por la menor pendiente de los suelos que a su vez favorece un mayor tiempo de persistencia de las grietas. Otra diferencia entre las dos zonas estudiadas es la menor tasa de infiltración final estable de Petrer $\left(13 \mathrm{~mm} \mathrm{~h}^{-1}\right)$. En Monnegre, $f c$ es de $21 \mathrm{~mm} \mathrm{~h}^{-1}$, lo que junto al retraso en la salida de la escorrentía da lugar a que después de 30 minutos (Vi30) el volumen de agua infiltrado fuera de $15 \mathrm{~mm}$ frente a los 27,5 precipitados. En Petrer, Vi30 es de $10 \mathrm{~mm}$. La variabilidad espacial de los procesos hidrológicos es mayor en Petrer que en Monnegre, con la excepción del parámetro Ce15, debido al retraso en la formación de la escorrentía en buena parte de las parcelas estudiadas (Tabla 3).

\subsection{Erodibilidad de los suelos, tasas de erosión y salinidad de la escorrentía}

Debido a las reducidas cubiertas vegetales y de piedras, y a la baja estabilidad de los agregados, la erodibilidad del suelo es muy elevada en los badlands (IMESON y VERSTRATEN, 1985; GERITS, 1991; CERDÀ, 1993c; en prensa b). El indicador más claro de esto es la concentración de sedimentos de la escorrentía, la cual alcanza los $42 \mathrm{~g}^{-1} \mathrm{l}^{-1}$ en Monnegre y 59 g. $~^{-1}$ en Petrer. Esto valores varían entre 20 y $62 \mathrm{~g} . ~^{1^{-1}}$ en Monnegre y entre 26 y 82 g. $1^{-1}$ en Petrer.

La tendencia de la concentración de los sedimentos (TCs) a lo largo del experimento en los suelos vegetados y con agregados estables presentan un continuo descenso debido al agotamiento de los sedimentos (ABRAHAMS et al., 1991; CERDÀ, 1993a). En los badlands el comportamiento es distinto: la concentración de sedimentos aumenta como indica el índice Tcs (Tabla 4, Fig. 2). En Monnegre el valor medio alcanza +0,43 aunque oscila entre $-0,25$ y $+1,13$. En este aspecto, también Petrer muestra un comportamiento más dinámico y el índice alcanza el valor $+0,56$, con variaciones entre $-0,06$ y $+1,21$. Es de destacar que en cuanto a la producción de sedimentos en los badlands, los cambios temporales durante un evento de lluvia de intensidad homogénea son mayores que las diferencias espaciales entre parcelas (ver Tabla 4 y Fig. 2). 
Tabla 4

PRINCIPALES PARÁMETROS EROSIVOS. CONCENTRACIÓN DE SEDIMENTOS

(CS), TENDENCIA DE LA CONCENTRACIÓN DE SEDIMENTOS (TCS), CONDUCTIVIDAD ELÉCTRICA DE LA ESCORRENTÍA (Sa), TENDENCIA DE CONDUCTIVI-

DAD ELÉCTRICA DE LA ESCORRENTÍA (Tsa) Y TASA DE EROSIÓN (Ter)

\begin{tabular}{|c|c|c|c|c|c|}
\hline Prueba & $\underset{\left(\mathrm{g} \mathrm{l}^{-1}\right)}{C s}$ & $T c s$ & $\begin{array}{c}S a \\
(\mu \mathrm{s})\end{array}$ & Tsa & $\begin{array}{c}\text { Ter } \\
\left(\mathrm{g} \mathrm{m}^{2} \mathbf{h}^{-1}\right)\end{array}$ \\
\hline \multicolumn{6}{|l|}{ Monnegre } \\
\hline 1 & 27,32 & $-0,07$ & 235 & $-5,29$ & 760,72 \\
\hline 2 & 42,49 & 0,33 & 313 & $-1,27$ & 940,47 \\
\hline 3 & 51,08 & 0,64 & 395 & $-6,77$ & 1108,87 \\
\hline 4 & 59,97 & 1,37 & 458 & $-8,87$ & 1350,13 \\
\hline 5 & 19,63 & $-0,15$ & 224 & $-4,69$ & 525,64 \\
\hline 6 & 56,00 & 1,13 & 403 & $-6,61$ & 1368,83 \\
\hline 7 & 62,27 & 1,09 & 481 & $-4,61$ & 1583,71 \\
\hline 8 & 33,09 & 0,12 & 215 & $-7,98$ & 761,43 \\
\hline 9 & 40,62 & $-0,25$ & 426 & $-7,68$ & 1004,51 \\
\hline 10 & 29,75 & 0,11 & 325 & $-6,99$ & 795,79 \\
\hline Media & 42,22 & 0,43 & 347,45 & $-6,08$ & 1020,01 \\
\hline std & 14,76 & 0,59 & 99,30 & 2,20 & 331,94 \\
\hline $\operatorname{cv}(\%)$ & 34,95 & 135,90 & 28,58 & $-36,13$ & 32,54 \\
\hline \multicolumn{6}{|l|}{ Petrer } \\
\hline 1 & 51,38 & $-0,04$ & 396 & $-5,35$ & 935,49 \\
\hline 2 & 72,46 & 1,21 & 553 & $-20,36$ & 2697,37 \\
\hline 3 & 60,47 & $-0,02$ & 436 & $-1,38$ & 1114,65 \\
\hline 4 & 25,75 & 0,36 & 215 & $-24,00$ & 847,85 \\
\hline 5 & 55,16 & 0,74 & 365 & $-12,54$ & 1991,47 \\
\hline 6 & 56,15 & 0,82 & 410 & $-10,45$ & 2136,59 \\
\hline 7 & 56,99 & 1,11 & 514 & $-13,12$ & 1655,53 \\
\hline 8 & 82,23 & $-0,06$ & 659 & $-19,89$ & 3220,57 \\
\hline 9 & 62,81 & 0,52 & 611 & $-21,75$ & 2454,69 \\
\hline 10 & 69,06 & 0,99 & 502 & $-15,42$ & 2283,83 \\
\hline Media & 59,24 & 0,56 & 466,01 & $-14,43$ & 1933,80 \\
\hline std & 15,01 & 0,49 & 129,72 & 7,34 & 788,91 \\
\hline cv $(\%)$ & 11,97 & 86,64 & 27,84 & $-50,89$ & 40,80 \\
\hline
\end{tabular}

La conductividad eléctrica de la escorrentía $(\mathrm{Sa})$ es un indicador directo de su salinidad y con ello de la magnitud de la exportación de solutos. En este aspecto, Petrer también es la zona donde se producen las mayores pérdidas; $416 \mu$ s frente a los $347 \mu$ s de Monnegre. Las variaciones espaciales son de 36 y $51 \%$ de $c v$ respectivamente para Petrer y Monnegre. En cuanto a la tendencia temporal hay un decrecimiento claro con oscilaciones entre $-1,38$ y -24 en Petrer, y entre $-1,27$ y $-8,87$ en Monnegre. En este aspecto las variaciones temporales también son mayores que las espaciales.

La tasa de erosión - dependiente de la concentración de sedimentos y del volumen de escorrentía - es mayor en Petrer $\left(1.934 \mathrm{~g} \mathrm{~m}^{2} \mathrm{~h}^{-1}\right)$ que en Monnegre $\left(1.020 \mathrm{~g} \mathrm{~m}^{2} \mathrm{~h}^{-1}\right)$. Las diferencias pueden oscilar entre valores ligeramente superiores a $500 \mathrm{~g} \mathrm{~m}^{2} \mathrm{~h}^{-1}$ en la parcela 5 de Monnegre a valores superiores a $3.000 \mathrm{~g} \mathrm{~m}^{2} \mathrm{~h}^{-1}$ en la parcela 8 de Petrer. Respecto a las variaciones espaciales, como en la mayoría de los aspectos, es Petrer la zona que muestra más diferencias entre parcelas (Tabla 4). 


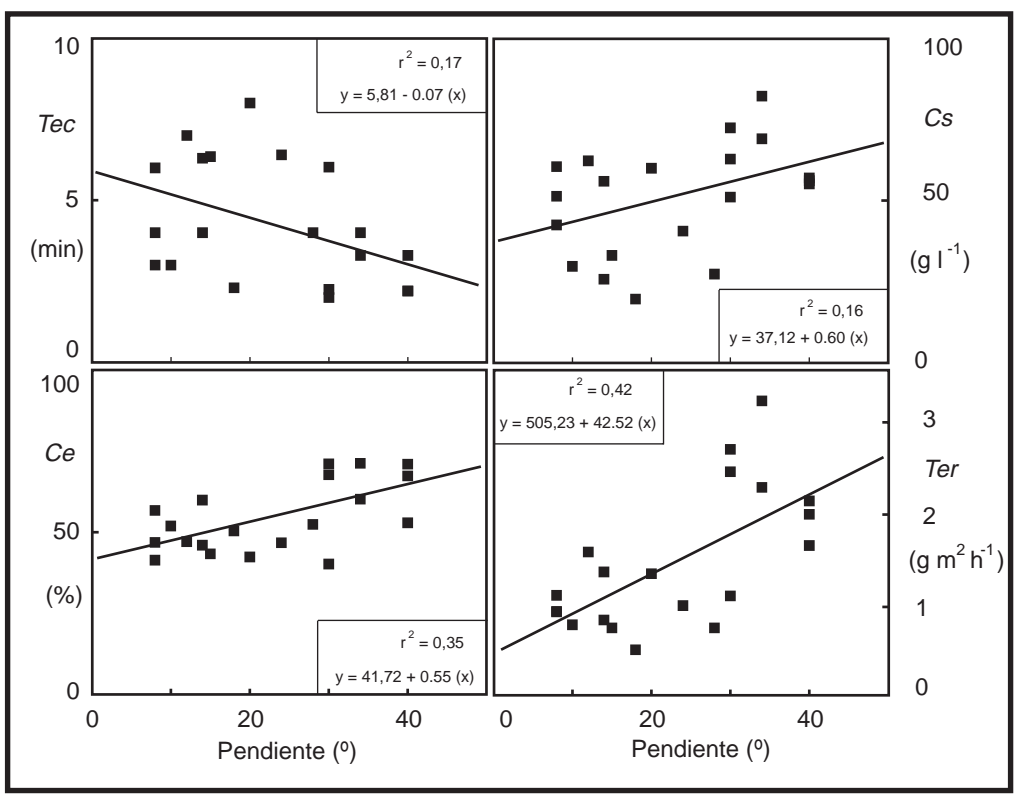

FIGURA 3. Relación entre la pendiente y el tiempo de llegada al colector de la escorrentía (Tec), el coeficiente de escorrentía (Ce), la concentración de sedimentos (Cs) y la tasa de erosión (Ter).

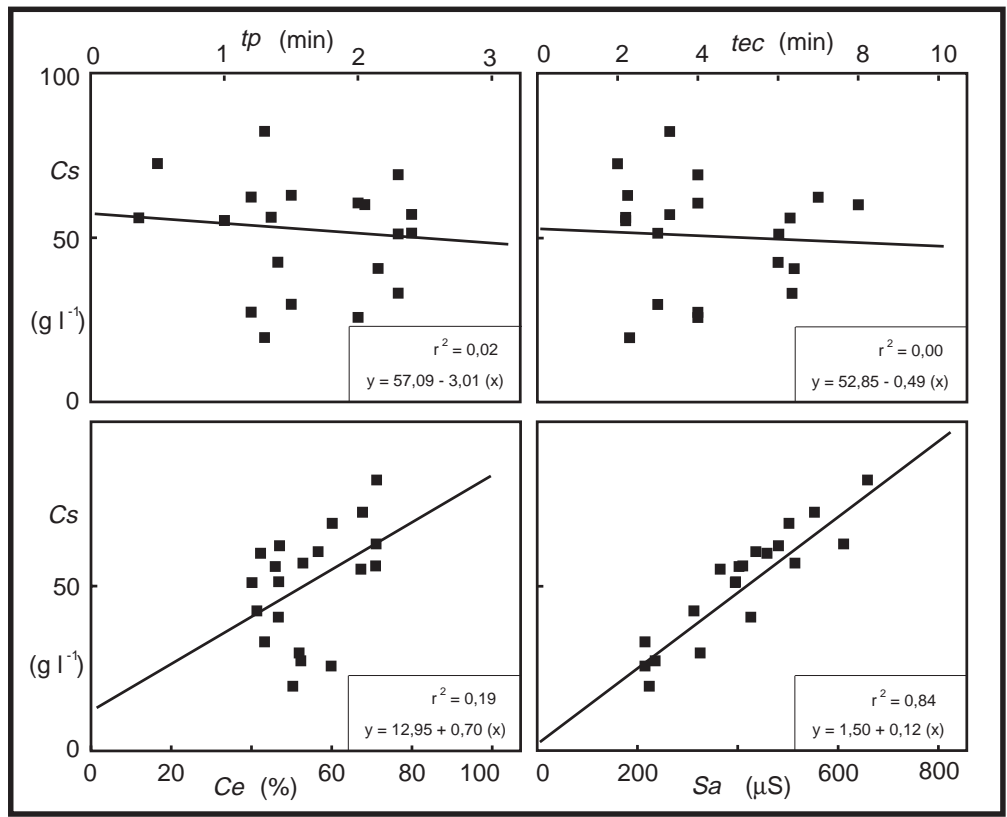

FIGURA 4. Relación entre distintos parámetros hidrológicos y erosivos con la concentración de sedimentos. Los parámetros hacen referencia al tiempo de inicio del encharcamiento (tp), tiempo de llegada de la escorrentía al colector (tec), coeficiente de escorrentía (Ce) y conductividad eléctrica de la escorrentía $(\mathrm{Sa})$. 


\subsection{Influencia de la pendiente}

La cubierta vegetal es el factor determinante de la producción de sedimentos como han demostrado distintos autores (ELWELL y STOCKING, 1976; LEE y SKOGERBOE, 1985; CERDÀ et al., 1995). En los ecosistemas semiáridos, el estrés hídrico da lugar a una menor biomasa y a la distribución de la vegetación en manchas, lo que favorece grandes contrastes dentro de una misma ladera (SÁNCHEZ y PUIGDEFÁBREGAS, 1994a; 1994b; CERDÀ, 1996c). En los badlands, el efecto de la vegetación es inexistente, y por ello otros factores gobiernan los procesos de erosión y sus tasas de actuación. Además de las diferencias litológicas, la pendiente es el factor determinante en la formación de la arroyada y las tasas de escorrentía y erosión. Como se presenta en la figura 3, el aumento de la pendiente favorece que la escorrentía llegue antes al colector (tec), que los volúmenes de escorrentía sean mayores $(\mathrm{Ce})$, que la escorrentía transporte más sedimentos debido a su mayor capacidad erosiva $(\mathrm{Cs})$ y que todo ello de lugar a tasas de erosión ( $T e$ ) mayores en los suelos con menores pendientes.

Otro factor de la erosión importante en medios semiáridos es la pedregosidad superficial (POESEN e INGELMO, 1992). Su reducida presencia en los badlands hace insignificante su influencia sobre las tasas de erosión. Las políticas de regeneración de los badlands deberán tener en cuenta la incorporación de vegetación alóctona o autóctona, la introducción de semillas (GARCÍA FAYOS y RECATALÀ, 1992; GARCÍA FAYOS et al., 1995) e incluso la introducción de fragmentos de roca que reduzcan las tasas de erosión y favorezcan suelos menos encostrados y más porosos (ABRAHAMS y PARSONS, 1991).

\subsection{Erodibilidad del suelo y procesos de erosión}

La erodibilidad — como característica del suelo— es una medida de la susceptibilidad al arranque y transporte por los agentes de la erosión. La erodibilidad del suelo es un efecto integrado de los procesos que regulan la absorción de la lluvia y la resistencia de las partículas del suelo al arranque y posterior transporte. Estos procesos están influidos por las propiedades del suelo tales como tamaño de partículas, estabilidad de agregados, materia orgánica, y por el tipo de arcillas o por características edáficas que afectan la estructura del suelo, la dispersión y la transmisión de agua (LAL, 1994). Hay pocos métodos válidos para la medición de la erodibilidad del suelo porque la mayoría reflejan una multitud de factores que no son intrínsecamente la erodibilidad. Las mediciones de la erodibilidad deben realizarse bajo condiciones conocidas para evitar cambios en la intensidad y volumen de la lluvia, o erosionabilidad (HUDSON, 1982). Las mediciones realizadas con los experimentos con lluvia simulada sobre los suelos desnudos de los badlands, permite entender la concentración de sedimentos de la escorrentía un parámetro definitorio de la erodibilidad de los suelos, al no existir la influencia de la vegetación.

En los badlands la erodibilidad es muy elevada como lo demuestran las altas concentraciones de sedimentos (Tabla 4), lo que ha sido confirmado en estudios que analizan la estabilidad de los agregados (IMESON y VERSTRATEN, 1989; CERDÀ, 1993c; 1996b). Comparado con otros suelos de la provincia de Alicante o de otras zonas del País Valenciano la magnitud de la erodibilidad de los suelos de los badlands es mucho mayor (CERDÀ, 1995b; 1996a). Ello es debido a la alta erodibilidad de los suelos y a que la escorrentía se genera rápidamente.

Los factores relacionados con la erodibilidad de los suelos se presentan en las figuras 4 y 5. Algunos de ellos (tp y tec) muestran una relación poco clara, aunque cuanto más pronto se produzca el encharcamiento y la escorrentía mayor será la concentración de 


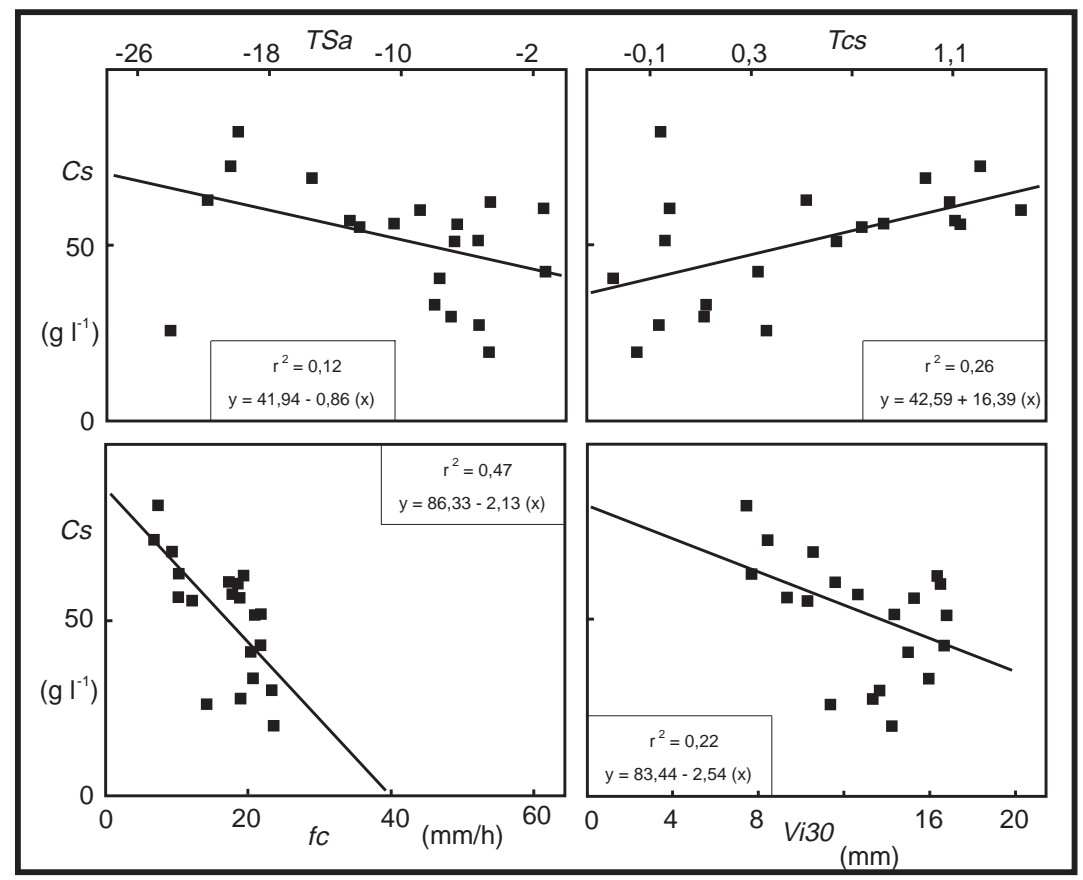

FiguRA 5. Relación entre distintos parámetros hidrológicos y erosivos con la concentración de sedimentos. Los parámetros hacen referencia a la tendencia de la conductividad eléctrica de la escorrentía (Tsa), tendencia de la concentración de sedimentos (Tcs), tasa de infiltración final estable (fc) y volumen de lluvia infiltrada después de 30 minutos (Vi30).

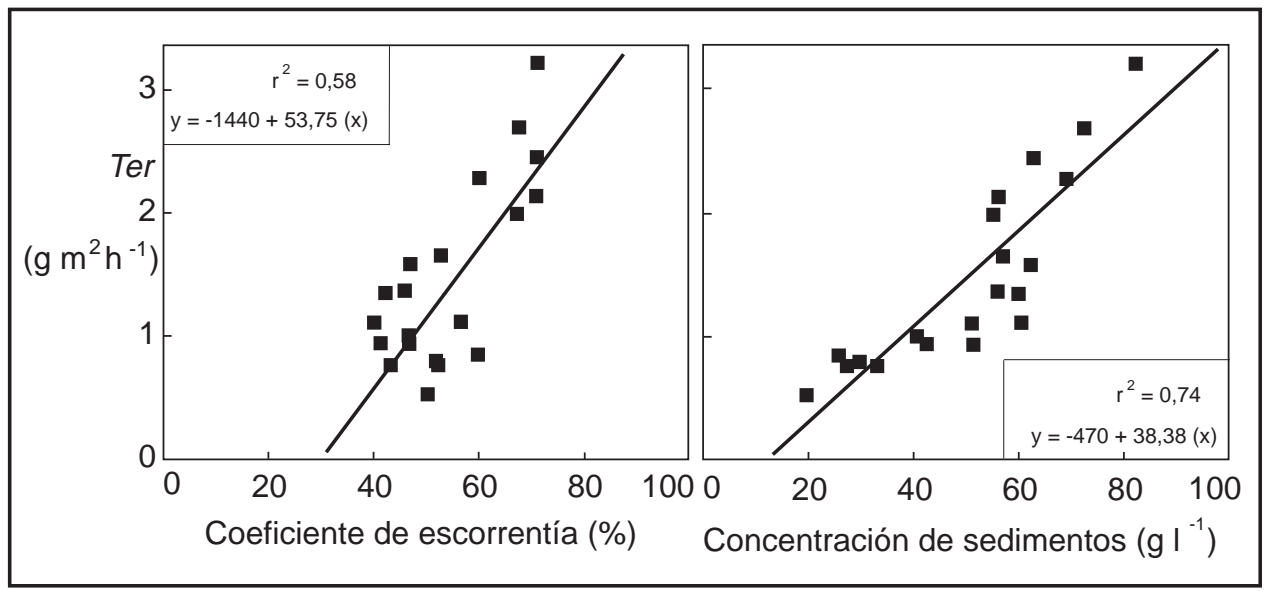

Figura 6. Relación entre el coeficiente de escorrentía y la concentración de sedimentos con la tasa de erosión (Ter). 
sedimentos en la escorrentía. Esto es debido a que la rotura de las costras y los agregados se produce antes y con ello el suministro de sedimentos a la arroyada superficial es mayor. Otros procesos hidrológicos presentan una relación muy clara con la concentración de sedimentos. Entre ellos cabe destacar la tasa de infiltración final estable $(f c)$, que se reduce cuando aumenta la $C s$, y el Coeficiente de escorrentía $(C e)$ que presenta una relación positiva con $C s$. Como $f c$, Vi30 presenta una relación negativa, lo que confirma la estudios previos en los que los suelos más erosionables son los que producen más escorrentía. Esto es contrario a lo encontrado en suelos vegetados en otras zonas del País Valenciano, donde la $C s$ decrece con el aumento de $C e$ (CERDÀ y NAVARRO, 1996). Esto es porque en los badlands el suministro de material es prácticamente inagotable debido a la dispersión de las agregados por el contacto con el agua y el impacto de las gotas de lluvia. En cambio, en los suelos vegetados, el proceso de erosión está determinado por el material disponible de ser erosionado, el cual decrece con la duración de la lluvia, al contrario que en los badlands (CERDÀ, 1993a). El control químico de la erosión en los badlands se ha puesto de manifiesto en distintos estudios (LARONNE, 1982; IMESON y VERSTRATEN, 1985; 1986; 1988). Este control químico del proceso de erosión en los badlands se ha confirmado aquí con la relación presentada en la figura 4 entre $S a$ y $C s$, ya que a mayor contenido de sales en la escorrentía se producen mayores concentraciones de sedimentos. El parámetro $T s a$ muestra una relación negativa con $C s$, lo que se debe a que el decrecimiento de la salinidad es mayor en los suelos más salinos, los cuales son también los más erosionables. En cambio, cuanto mayor es Tcs mayor es $C s$, lo que significa que las parcelas más erosionables muestran una tendencia a serlo más cuando se prolongan los chubascos. Ello favorecerá diferencias entre las parcelas en las tasas de erosión mucho mayores durante eventos de larga duración.

\subsection{Tasas de erosión}

Dos son los factores inmediatos que se consideran determinantes de las tasas de erosión producidas por la arroyada superficial: el caudal de la escorrentía (lluvia que se transforma en escorrentía al no infiltrarse) y la concentración de sedimentos en la escorrentía (partículas de suelo en el agua). En los badlands, ambos factores son determinantes para la explicación de las tasas de erosión (Fig. 6). Pero los badlands destacan por la magnitud de las tasas de erosión incluso a escala entre regueros donde la arroyada superficial es difusa y su capacidad de remoción de sedimentos es menor que en superficies mayores, donde la formación de regueros y cárcavas favorecen tasas de erosión mayores (MORGAN, 1986). Estas elevadas tasas de erosión son debidas a que el 54\% de la lluvia se transforma en escorrentía superficial y a que ésta transporta en término media más de $50 \mathrm{~g} \mathrm{l}^{-1}$.

Las diferencias entre parcelas, además de por la pendiente, se deben a las diferencias litológicas (minerales de arcillas, sales, etc. distintos) que como se ha demostrado en otros trabajos determinan los procesos de erosión y sus tasas, así como las formas superficiales (CALVO et al., 1991; GERITS, 1991; PAYÀ y CERDÀ, 1992). La influencia del roquedo también explica las menores tasas de erosión y escorrentía en los badlands desarrollados sobre arcillas del Keuper (CERDÀ y PAYÀ, 1995; CERDÀ, 1996d; CERDÀ, en preparación).

\section{Conclusiones}

Las laderas de los badlands están dominadas por procesos superficiales muy activos. La escorrentía superficial directa se produce con gran rapidez y dan lugar a volúmenes muy 
elevados. Chaparrones de $55 \mathrm{~mm} \mathrm{~h}^{-1}$ durante 40 minutos sobre suelos muy secos —situación típica de verano- generan escorrentías elevadas (54\% de media) y concentraciones de sedimentos muy altas (> 50\%). El que las arroyadas sean tan fuertes y la erodibilidad del suelo tan alta da lugar a la formación de regueros en distancias muy cortas. La dinámica erosiva de los badlands impide la revegetación de los suelos al erosionarse con gran facilidad la regolita, con lo que la formación de un suelo capaz de soportar una cubierta vegetal estable es prácticamente imposible mediante procesos naturales y a corto plazo. Si bien la inexistencia de vegetación y de fragmentos de roca determinan tasas de erosión muy altas, el control de procesos de erosión dentro de los badlands está relacionado con la pendiente y las reacciones químicas que se producen al entrar en contacto el agua con el suelo. Por ello, las diferencias litológicas en los badlands determinan los procesos de erosión por arroyada superficial y sus tasas de actuación. Estos factores - roquedo y pendiente- explican las variaciones dentro de los badlands, mientras que la falta de cubierta vegetal y de piedras son las causas de las altas tasas de erosión que los diferencia del resto de paisajes del territorio alicantino.

\section{Agradecimientos}

Agradecemos a Mario Payà y a toda su familia la ayuda prestada durante la realización del trabajo de campo, a la Comisión Interministerial de Ciencia y Tecnología la financiación del trabajo de campo (proyectos AMB93-0844-C06-04 y NAT91-0741) y al Ministerio de Educación y Ciencia la concesión de una beca de Perfeccionamiento de Doctores y Tecnólogos en el Extranjero al primero de los autores.

\section{Bibliografía}

ABRAHAMS, A.D. y PARSONS, A.J. (1991): Relation between infiltration and stone cover on a semiarid hillslope, Southern Arizona. Journal of Hydrology, 122, 49-59.

ABRAHAMS, A.D. y PARSONS, A.J. (1994): Geomorphology of Desert environments. Chapman and Hall, Cambridge, 674 pp.

ABRAHAMS, A.D.; PARSONS y LUK, S.H. (1988): Hydrologic and sediment response to simulated rainfall on desert hillslopes in southern Arizona. Catena, 15, 103-117.

ABRAHAMS, A.D.; PARSONS y LUK, S.H. (1991): The effect of spatial variability in overland flow on the downslope of soil loss on semiarid hillslope, Southern Arizona. Catena, 18, 255-270.

BRYAN, R. y YAIR, A. (1982): Badland geomorphology and piping. Goebooks, Norwich, 409 pp.

CALVO, A.; HARVEY, A.M. y PAYÀ, J. (1991): Process interactions and badland development in SE Spain. En SALA, M.; RUBIO, J.L. y GARCÍA-RUIZ, J.M. (Eds.): Soil Erosion Studies in Spain, 75-96.

CALVO, A.; HARVEY, A. y PAYÀ, J. (1992): Response of badland surfaces in south east Spain to simulated rainfall. Cuaternario y Geomorfología, 6, 3-14.

CAMPBELL, I.A. (1974): Erosion rates in the Steveville badlands Alberta. Z. Geomorph. N. F. Suppl.-Bd., 21, 122-137.

CAMPBELL, I.A. (1989): Badland and Badland Gullies. En THOMAS, D.S.G. (Ed.): Arid Zone geomorphology, 159-186.

CERDÀ, A. (1993a): La infiltración en los suelos del País Valenciano. Factores y variaciones espacio-temporales. Tesis Doctoral Inédita. Universitat de València. 357 pp. + apéndices.

CERDÀ, A. (1993b): Metodología para el estudio de la hidrología y erosión de superficies degradadas (badland) a partir de lluvia simulada. Cuaternario y Geomorfología, 7, 35-48.

CERDÀ, A. (1993c): Estabilidad de agregados en suelos degradados. País Valenciano. Nuevos Procesos Territoriales, 187-192.

CERDÀ, A. (1995a): Factores y variaciones espacio-temporales de la infiltración en los ecosistemas mediterráneos. Editorial Geoforma. Logroño, 151 pp. 
CERDÀ, A. (1995b): Hidrología y erosionabilidad de los suelos en ambientes semi-áridos, Petrer, Alicante. Studia Oecologica, 12, 159-164.

CERDÀ, A. (1996a): Régimen hídrico de los suelos en zonas acarcavadas. Algunos ejemplos en la cuenca experimental de Petrer. Alicante. IV Congreso Nacional de Geomorfología, en prensa.

CERDÀ, A. (1996b): Soil aggregate stability in three Mediterranean environments. Soil technology, en prensa.

CERDA, A. (1996c): The effect of patchy distribution of Stipa tenacissima L. on runoff and erosion. Journal of Arid Environments, en prensa.

CERDÀ, A. (1996d): Influencia del roquedo sobre los procesos de erosión en badlands. Edafología, en revisión.

CERDÀ, A. (en preparación): Influencia de la litología sobre los procesos de erosión en los badlands del Ríu Monnegre.

CERDÀ, A. y GARCÍA-FAYOS, P. (1996b): The influence of slope angle on sediment, water and seed losses on badland landscapes. Geomorphology, en prensa.

CERDÀ, A. y GARCÍA-FAYOS, P. (1996a): Relaciones entre las pérdidas de agua, suelo y semillas en zonas acarcavadas. Influencia de la pendiente. Cuadernos de Investigación Geográfica. En prensa.

CERDÀ, A. y NAVARRO, R. (1996): Tasas de erosión en una solana en la Serra Grossa. La Costera. Cuadernos de Geografía, en prensa.

CERDÀ, A. y PAYÀ, J. (1995): Morfología y procesos superficiales en badlands sobre arcillas del Keuper, Requena, Valencia. Cuaternario y Geomorfología, 9, 59-72.

CERDÀ, A.; CALVO, A.; SORIANO, M.D.; BOIX, C. e IMESON, A. (1995): Escorrentía y movilización de suelo en ambiente semiárido. Influencia de la cubierta vegetal. En Actas del XX Reunión Nacional de Suelos: Degradación y conservación de suelos en áreas de montaña, 109-117.

COSTA, M. (1986): La vegetación en el País Valenciano. Universitat de València. Servicio de Publicaciones.

ELÍAS CASTILLO, J. y RUIZ BELTRÁN, L. (1977): Precipitaciones máximas en España. Ministerio de Agricultura, Madrid, 545 pp.

ELWELL, H.A. y STOCKING, M.A. (1976): Vegetal cover to estimate soil erosion hazard in Rodhesia. Geoderma, 15, 61-70.

GARCÍA FAYOS, P. y RECATALÀ, R.M. (1992): La reserva de semillas en una cuenca de «badlands» (Petrer, Alicante). Pirineos, 140, 29-36.

GARCÍA FAYOS, P.; RECATALÀ, T.M.; CERDÀ, A. y CALVO, A. (1995): Seed population dynamics on badland slopes in SE Spain. Journal of Vegetation Science, 6, 691-696.

GEIGER, F. (1970): Die Ariditat in Sudostspanien. Stuttgarter Geographische Studien, Band 77.

GERITS, J.J.P. (1991): Physico-chemical tresholds for sediment detachment, transport and deposition. Universiteit van Amsterdam, Amsterdam, $186 \mathrm{pp}$.

GREGORY, K.J. y WALLING, D.E. (1973): Drainage basins forms and processes: a geomorphological approach. Arnold, London, $458 \mathrm{pp}$.

HARVEY, A.M. y CALVO, A. (1989): Distribution of badlands in Southeast Spain: implications of climatic change. En IMESON, A.C. and De GROOT, R.S. (Eds.): Landscape-ecological Impact of Climatic Change, $14 \mathrm{pp}$.

HORTON, R.E. (1940): An approach toward a physical interpretation of infiltration capacity. Proc. Soil Sci. Soc. Am., 5, 399-417.

HOWARD, A.D. (1994): Badlands. En ABRAHAMS, A.D. y PARSONS, A.J. (Eds.): Geomorphology of Desert Environments, 213-242.

HUDSON, N. (1982): Conservación de suelo. Reverté, S. A. Barcelona, 335 pp.

IGME (1978a): Mapa Geológico de España (E:1/50.000). ELDA (Hoja 871).

IGME (1978b): Mapa Geológico de España (E:1/50.000). ALICANTE (Hoja 872).

IMESON, A.C. y VERSTRATEN, J.M. (1985): The erodibility of highly calcareos soil material from Southern Spain. Catena, 12, 291-306.

IMESON, A.C. y VERSTRATEN, J.M. (1986): Erosion and sediment generation in semiarid and Mediterranean environments: the response of soils to wetting by rainfall. Journal of Water Resources, 5 (1) 388-417. 
IMESON, A.C. y VERSTRATEN, J.M. (1988): Rills on badland slopes: a physico-chemical controlled phenomenon. Catena suppl.-Bd., 12. 139-53.

IMESON, A.C. y VERSTRATEN, J.M. (1989): The microaggregation and erodibility of some semiarid and mediterranean soil. Catena supplement, 14, 11-24.

IMESON, A.C.; LAVEE, H.; CALVO, A. y CERDÀ, A. (1996): The erosional response of calcareous soils along a climatological gradient in southeast Spain. Geomorphology, en prensa.

LAL, R. (1994): Soil Erosion Research Methods. Soil and Water Conservation Society (Ankeny), $3^{\text {th }}$ edition, St. Lucie press, Delray Beach, $340 \mathrm{pp}$.

LARONNE, J. (1982): Sediment and solute yield from Mancos Shale hillslopes. Colorado and Utah. En BRYAN, R. y YAIR, A. (Eds.): Badland Geomorphology and Piping, 181-194.

LEE, C.R. y SKOGERBOE, J.G. (1985): Quantification of erosion control by vegetation on problem soils. En EL SWAIFY, W.C.; MOLDENHAUER, W.C. y LO, A. (Eds.): Soil Erosion and Conservation, 437-444.

LÓPEZ BERMÚDEZ, F. y ALBADALEJO, J. (1990): Factores ambientales de la degradación del suelo en el área mediterránea. En ALBADALEJO, J.; STOCKING, M.A. y DÍAZ, E. (Eds.): Degradación y regeneración del suelo en condiciones ambientales mediterráneas, 15-45.

MATARREDONA, E. y MARCO, J.A. (1991): El relieve y los suelos. Atlas Temático de la Comunidad Valenciana, 41-60.

MEYER, L.D. (1994): Rainfall simulators for soil erosion research. En LAL, R. (Ed.): Soil Erosion Research Methods, 83-103. Soil and Water Conservation Society (Ankeny), $3^{\text {th }}$ edition, St. Lucie press, Delray Beach, 340 pp.

MORGAN, R.P.C. (1986): Soil Erosion and Conservation. Longman, New York, 298 pp.

NAVAS, A. (1988): La participación de los yesos en la salinización de las aguas superficiales de la Cuenca del Ebro. Tesis Doctoral inedita. Universidad de Zaragoza, 444 pp.

PAYÀ, J. y CERDÀ, A. (1992): Cambios morfológicos y respuesta a la lluvia simulada de tres superficies de Badland. Petrer. Alacant. En LÓPEZ BERMÚDEZ, F.; CONESA GARCÍA, C. y ROMERO DÍAZ, M.A. (Eds.): Estudios de Geomorfología en España, 161-170.

PÉREZ CUEVA, A. J. (1994): Atlas climático de la Comunidad Valenciana. Generalitat Valenciana, València, $205 \mathrm{pp}$.

POESEN, J. e INGELMO SÁNCHEZ, F. (1992): Runoff and sediment yields from topsoils with different porosity as affected by rock fragment cover and position. Catena, 19, 451-474.

PUIGDEFÁBREGAS, J. (1988): Erosión y desertificación en España. El Campo, 132, 63-83.

RODRÍGUEZ AIZPEOLEA, J. (1992): Un ejemplo de la influencia del uso y abandono de bancales de fondo de canal en la evolución de Badlands (Petrer-Alacant). En LÓPEZ BERMÚDEZ, F.; CONESA GARCÍA, C. y ROMERO DÍAZ, M.A. (Eds.): Estudios de Geomorfología en España, 211-220.

SÁNCHEZ, G. y PUIGDEFÁBREGAS, J. (1994a): Interactions of plant growth and sediment movement on slopes in a semi-arid environment. Geomorphology, 9, 243-260.

SÁNCHEZ, G. y PUIGDEFÁBREGAS, J. (1994b): Simulación del desarrollo de macollas de esparto (Stipa tenacissima L.) en relación a la arquitectura y el transporte de sedimentos. Studia Oecologica, X-XI, 211-219.

SAVAT, J. (1981): Work done by splash: laboratory experiments. Earth Surfaces Processes and Landforms, 6, 275-283.

THORNES, J.B. (1976): Semi-arid erosion systems: case studies from Spain. Geogr. Papers, $\mathrm{n}^{\circ} 7$, London School of Economics. 